Intraoperative use of analgesics was significantly reduced. Epidural analgesia was continued for 2 days postoperatively.

Conclusions Despite various challenges, this case of severe kyphoscoliosis was successfully managed with fluoroscopyguided epidural anaesthesia. Fluoroscopy is a well-established imaging modality and its knowledge adds to the Anaesthesiologist armamentarium and offers an opportunity to provide neuraxial anaesthesia in patients with anticipated difficulty.

\section{CONTINUOUS ERECTOR SPINAE PLANE BLOCK AS MULTIMODAL ANALGESIA AND INTRAOPERATIVE HEMODYNAMIC STABILITY IN OPEN NEPHRECTOMY FOR LIVING DONOR KIDNEY TRANSPLANT}

R Rahmi* ${ }^{*}$ RB Sukmono. Universitas Indonesia, Jakarta, Indonesia

\subsection{6/rapm-2021-ESRA.13}

Background and Aims Open nephrectomy is associated with moderate to severe postoperative pain.1 Erector spinae plane block (ESPB) provides effective analgesia for various surgical procedure but only a few reports on ESPB used for pain control in open nephrectomy.2 We reported two cases of

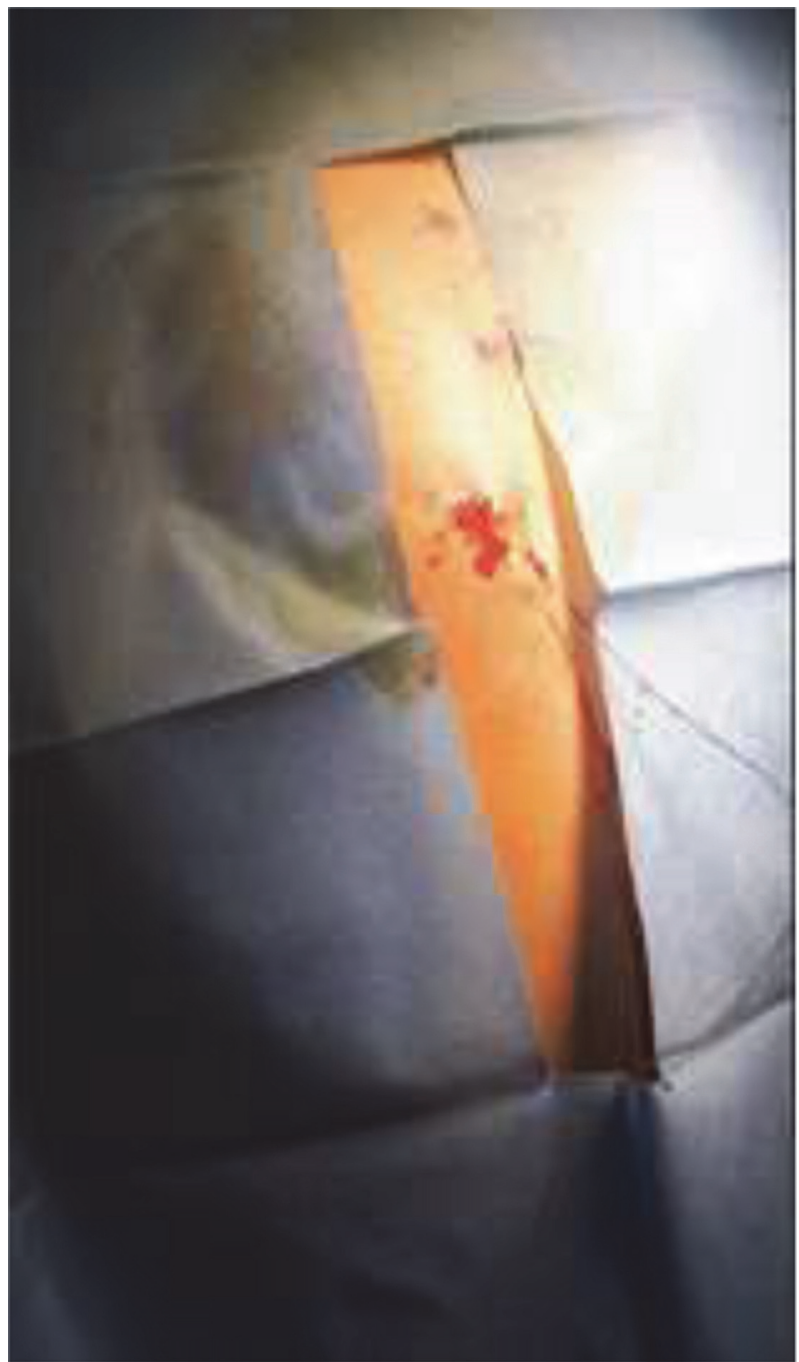

Abstract 13 Figure 1 continuous ESPB as multimodal analgesia and intraoperative hemodynamic stability in open nephrectomy for living donor kidney transplant.

Methods Two adult patients underwent open nephrectomy for living donor renal transplant. ESPB was performed before general anesthesia with ultrasound guided using high frequency linear probe at level of T8-T9. After transversus process and erector spinae muscle were identified, hydro dissection with $2 \mathrm{ml}$ of normal saline was used to confirm the correct needle tip position. 18-gauge epidural catheter then inserted into the plane between erector spinae and transverse process. $20 \mathrm{ml}$ local anesthetic 0,25\% levobupivacaine was injected and spread of local anesthetic under erector spinae muscle was seen by ultrasound. For postoperative pain control, patients were administered $10 \mathrm{ml}$ levobupivacaine $0,125 \%$ via catheter at the end of surgery, continuous levobupivacaine $0,125 \% 6$

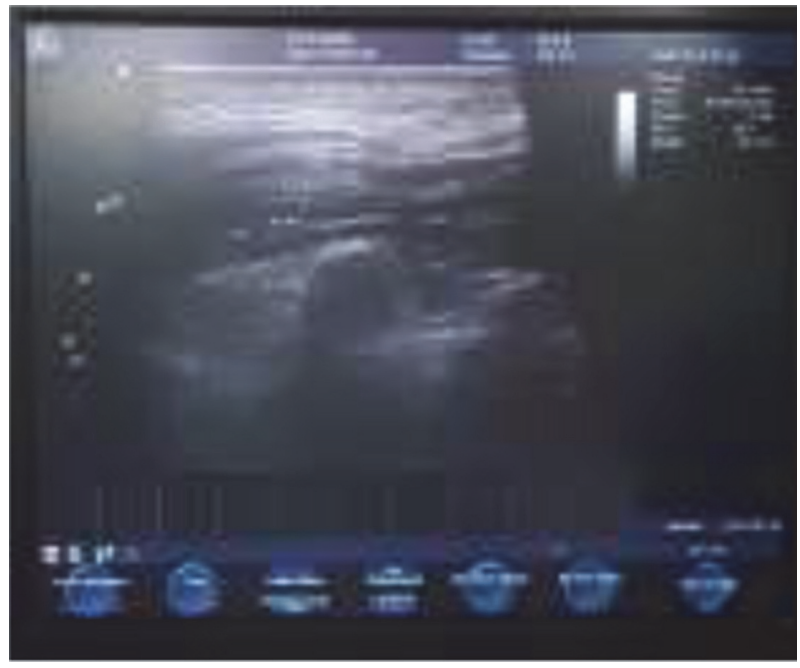

Abstract 13 Figure 2

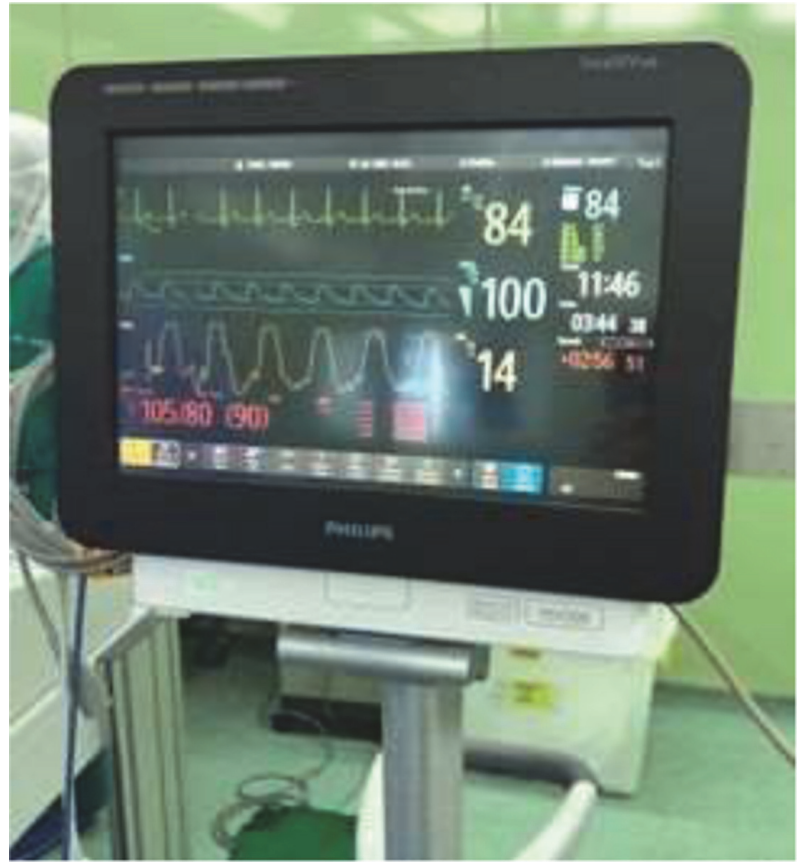

Abstract 13 Figure 3 
$\mathrm{ml} /$ hour and paracetamol $1 \mathrm{gr} / 8$ hours. Vital sign was recorded intraoperatively. NRS was measured at recovery room, 6, 12, 24 hours postoperatively.

Results Hemodynamic instability was not observed during incision and intraoperatively. NRS was 2 in recovery room and ward. No additional opioid used intraoperatively.

Conclusions Continuous ESPB is effective to reduce pain, total opioid consumption and can be used as alternative for epidural analgesia. Prospective trials are needed to evaluate the efficacy of continuous ESPB for open nephrectomy.

\section{SUPERIOR TRUNK BLOCK AND SUPERFICIAL CERVICAL PLEXUS IN PATIENT WITH DIFFICULT AIRWAY FOR CLAVICULA SURGERY: A MANAGEMENT OPTION}

JC Delgado Marquez*, G Hernandez Rodriguez. Hospital General de Queretaro, Santiago de Queretaro, Mexico

\subsection{6/rapm-2021-ESRA. 14}

Background and Aims There are different scales to assess the degree of difficulty to ventilate and intubate the different patients. unfortunately there is the possibility of having an unanticipated difficult airway scenario. In this case, a 71-year-

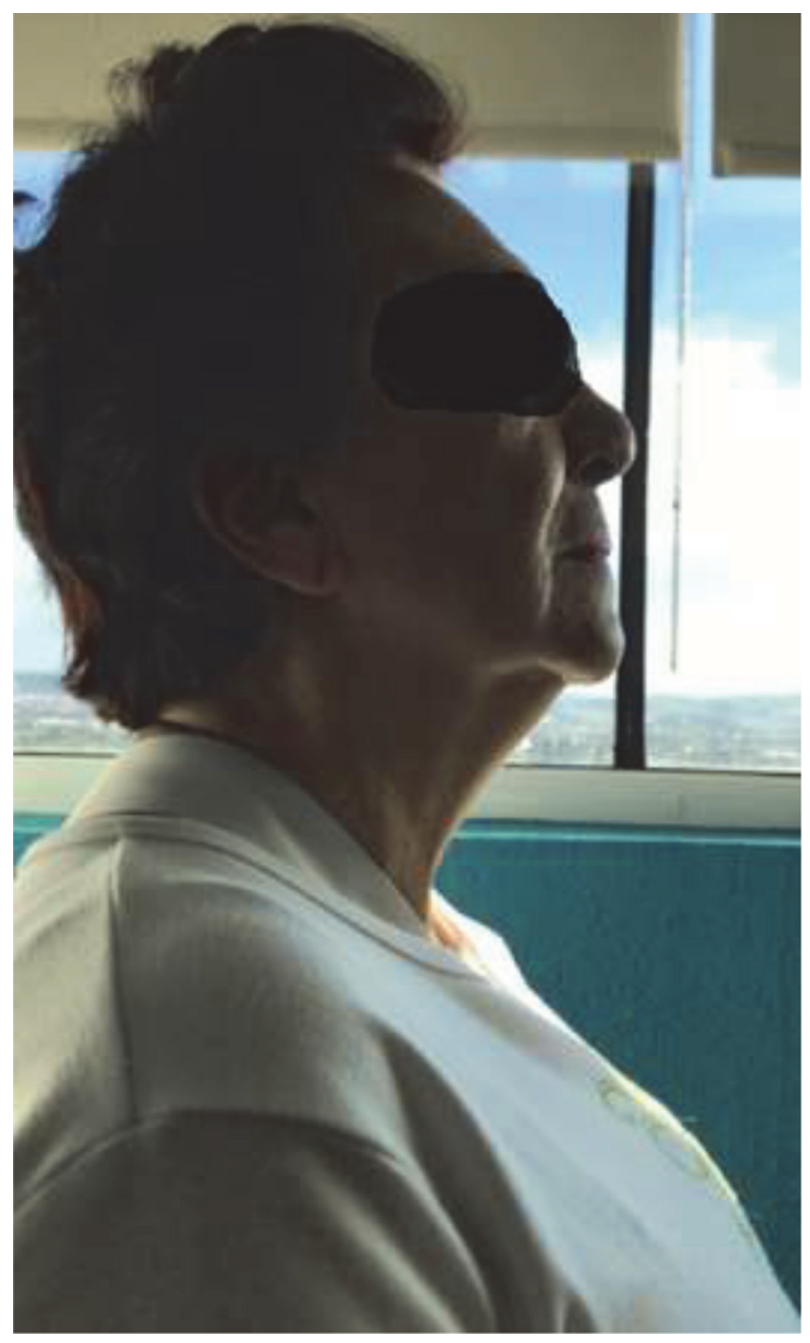

Abstract 14 Figure 1

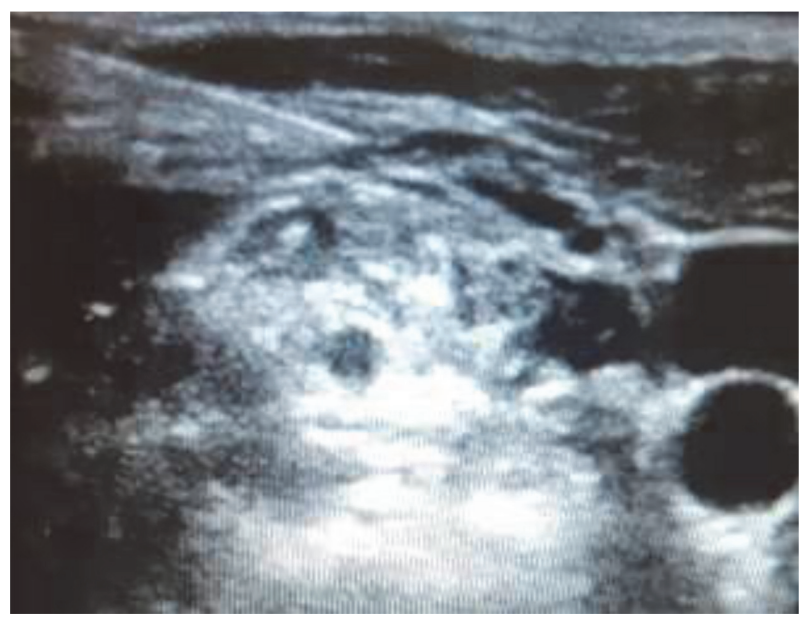

Abstract 14 Figure 2

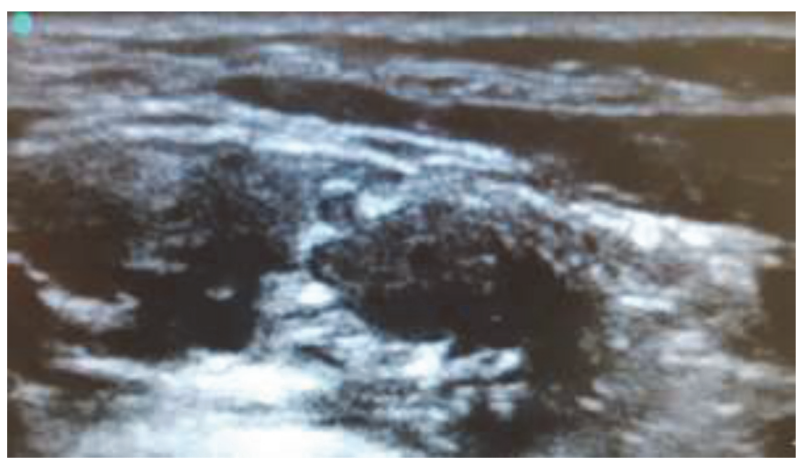

Abstract 14 Figure 3

old female patient with chronic arterial hypertension managed with a diuretic and calcium antagonist; hypothyroidism treated with levothyroxine 100 milligrams a day. His current condition begins with a fall from the stairs with pain in his left shoulder, with deformity and inability to move his upper limb. She has a history of difficult intubation, in her last surgery she required advanced CPR secondary to hypoxia. Therefore, this patient represents a challenge.

The objective is to demonstrate the usefulness and advantages offered by this regional technique.

Methods An ultrasound-guided block was performed with a $50 \mathrm{~mm}$ needle with 20 gauge in the superficial cervical plexus and upper trunk of the brachial plexus in plane, with doses of ropivacaine at $0.25 \%$ and lidocaine with epinephrine at $1.75 \%$ with a volume of 10 and 5 milliliters, respectively without complications.

Results The combined locoregional blockade in this patient was satisfactory, allowing the surgery to be performed with the patient awake, avoiding compromise and manipulation of the airway.

Conclusions The anesthetic technique of choice in this type of patient is controversial, but the use of resources and alternatives that can be used is important to reduce the risks and possible complications associated with unwanted events during surgery. It is important to individualize the cases and assess the risk benefit of each anesthetic technique. 